\title{
Competencias parentales percibidas y calidad de vida
}

\author{
ALFONSO URZÚA M. ${ }^{1}$, JAVIER GODOY B. ${ }^{1}$, KARLA OCAYO T. ${ }^{1}$ \\ 1. Área Psicología de la Salud, Escuela de Psicología, Universidad Católica del Norte, Antofagasta, Chile.
}

\begin{abstract}
Perceived parental competencies and quality of life

Introduction: Comprehensive assessment measures in non-diseased population, requires the study of quality of life and factors related to it. Objective: To analyze the relationship between self-reports of parental competencies and quality of life. Methods: 1,130 chilean children and young between 8 and 18 years of public, subsidized and private school, answered the Quality of Life questionnaire KIDSCREEN-52 and the test for parenting skills ECPP-h. Results: We found differences in quality of life and perceived parental competences by sex and type of school. Parental involvement in scholar issues and parental control are linked to various domains of quality of life, independent of age. Conclusion: Parental involvement in school issues promotes academic success and is perceived by children as positive in their assessment of well being. This information can be used for possible contingency plans in order to improve quality of life of children and adolescents.
\end{abstract}

(Key words: Quality of life, parenting skills, childhood, adolescence).

Rev Chil Pediatr 2011; 82 (4): 300-310

\section{RESUMEN}

Introducción: La necesidad de incorporar medidas de evaluación integrales en población no enferma, amerita el estudio de la calidad de vida y factores relacionados a ésta. Objetivo: Bajo un diseño transversal, se describe y analiza la relación existente entre los auto-reportes de competencias parentales y calidad de vida. Método: Se evalúa a 1130 niños/as y adolescentes chilenos entre 8 y 18 años de establecimientos públicos, subvencionados y privados, a través del cuestionario de Calidad de Vida KIDSCREEN-52 y el cuestionario de Competencias Parentales ECPP-h. Resultados: Se observan diferencias en las dimensiones de calidad de vida y en las competencias parentales percibidas dadas por el sexo y el tipo de establecimiento educacional. La implicancia escolar y el control parental se hallan vinculados a diversos dominios de la calidad de vida,

Trabajo recibido el 08 de marzo de 2011, devuelto para corregir el 18 de abril de 2011, segunda versión el 06 de mayo de 2011, aceptado para publicación el 16 de mayo de 2011.

Correspondencia a:

Dr. Alfonso Urzúa M.

E-mail:alurzua@ucn.cl 
independientemente del rango de edad. Conclusión: El involucramiento de los padres en asuntos escolares de niños/as y adolescentes promueve el éxito escolar y es percibido por los menores como positivo en cuanto su evaluación de bienestar, aportando información que puede ser utilizada para posibles planes de intervención con el objeto de mejorar la calidad de vida de los niños/as y adolescentes.

(Palabras clave: Calidad de vida, competencias parentales, niñez, adolescencia).

Rev Chil Pediatr 2011; 82 (4): 300-310

\section{Introducción}

La Organización Mundial de la Salud define la Calidad de Vida (CV) como la percepción individual de la posición en la vida en el contexto de la cultura y sistema de valores en el cual se vive y su relación con las metas, expectativas, estándares e intereses ${ }^{1}$. En los infantes y adolescentes, incorpora la percepción de éstos sobre su bienestar físico, psicológico y social, dentro del contexto cultural especifico, desarrollo evolutivo y diferencias individuales ${ }^{2}$.

Pese a la importancia adquirida como medida de resultados en salud y bienestar en la infancia y la adolescencia, prevalece la investigación en $\mathrm{CV}$ relacionada con la salud (CVRS), vinculada a la salud física y mental, discapacidad ${ }^{3-6}$ y creación y adaptación de instrumentos de medidas específicos ${ }^{7,8}$. El foco ha estado centrado en el reporte de la CV perteneciente a población con enfermedades crónicas, permitiendo conocer el estado de salud e identificar los niveles de morbilidad, valorar el impacto de los tratamientos sobre la salud individual y grupal y facilitar el proceso de toma de decisiones clínicas ${ }^{9-11}$.

En los últimos años se ha intentado ampliar el espectro de estudios desde la CVRS a la medición de la CV general, a fin de conocer la percepción de satisfacción y bienestar de los menores no enfermos, en distintas dimensiones de su vida diaria. Esto se realiza a través de cuestionarios específicos que recogen la evaluación de los padres o cuidadores, o bien, y cada vez más frecuentemente, la autopercepción de los participantes. Esto difiere a lo que sucedía hace unos años, donde preponderaba la opinión de los padres con respecto a la CV de los menores ${ }^{12}$.
Pese a esto, no existen muchas investigaciones que informen acerca de qué factores inciden en la CV de niños/as y adolescentes. Estudios realizados en Chile en población escolar, evidencian que diversos factores como nivel socioeconómico, salud física y la relación con la familia y los padres influyen en una baja o alta CV percibida en esta población ${ }^{11,13}$, siendo este último factor uno de los que a pesar de la importancia detectada no presenta mayores desarrollos como línea de investigación.

El bienestar infantil puede ser consecuencia del predominio de experiencias de buen trato que un niño/a tiene. Estos buenos tratos no sólo son los que los padres son capaces de ofrecer, sino también el resultado de los recursos que la sociedad dispone para garantizar la satisfacción de las necesidades infantiles y el respeto de sus derechos, así como para apoyar y favorecer el desarrollo de estrategias y habilidades parentales que favorezcan este buen trato y así responder apropiadamente a las necesidades del desarrollo del niño/a $\mathrm{a}^{14}$.

Las estrategias y habilidades, denominadas Competencias Parentales, pueden ser definidas como el conjunto de capacidades que permiten a los padres afrontar de modo flexible y adaptativo su rol, de acuerdo con las necesidades evolutivas y educativas de sus hijos y bajo los estándares considerados como aceptables por la sociedad, aprovechando todas las oportunidades y apoyos que les brindan los sistemas de influencia de la familia para desplegar dichas capacidades ${ }^{15}$.

Para Barudy ${ }^{14}$, es una forma para referirse a las capacidades y prácticas para cuidar, proteger y educar a los hijos y de esa manera asegurarles un desarrollo sano. Las competencias parentales cumplen un papel fundamental en la crianza y bienestar de los hijos/as, ya que 
son estas herramientas las principales (y a veces las únicas) que cuentan para sostener el cuidado afectivo y material que los niños/as requieren para su desarrollo evolutivo y social, sin olvidar las demandas del entorno en donde se desenvuelven cotidianamente.

Pese a esta reconocida importancia, son escasas las investigaciones que analizan la influencia, análisis y la evaluación de las competencias parentales en un plano no jurídico, al igual que las que las relacionan con su impacto en la CV y el bienestar de los menores. En este contexto, esta investigación tiene por objetivo describir y analizar la relación existente entre el autoreporte de la CV en niños/ as y adolescentes y la percepción que tienen éstos sobre las competencias parentales de sus padres. Aun cuando no se ha encontrado evidencia empírica al respecto, se considera como hipótesis de investigación que a mayor competencia parental percibida, mejor será el autoreporte de la CV.

\section{Pacientes y Método}

Utilizamos un muestreo intencionado, para obtener cuotas similares en cuanto sexo, edad y tipo de establecimiento educacional de menores provenientes de Establecimientos educacionales privados, subvencionados (privados con aporte estatal) y municipalizados (públicos) de la ciudad de Antofagasta.

Para evaluar la CV se utilizó la versión de autoreporte del Cuestionario Kidscreen-52, el cual presenta buenas propiedades psicométricas $^{16,17}$. Este cuestionario valora subjetivamente la salud y el bienestar a través de 10 dimensiones de CVRS: Bienestar físico (BIEFIS), que a través de 5 preguntas recoge los niveles de actividad física, energía y buena condición física del chico/a; Bienestar psicológico (BIEPSI), que en 6 ítems recoge el bienestar psicológico del chico/a, incluidas las emociones positivas y la satisfacción con la vida; Estado de ánimo (EANIM) el cual a través de 7 preguntas indaga en las experiencias negativas, los estados de ánimo depresivos y sensaciones de estrés; Autopercepción (APERC), que con 5 ítems evalúa la percepción del chico/a sobre sí mismo/a, la apariencia física y la satisfacción relacionada con ellos; Autonomía (AUTON) la cual en 5 preguntas recoge las oportunidades de disponer de su tiempo de ocio; Relación con los padres y vida familiar (REPAD), que con base a 6 ítems examina la relación con los padres y la atmósfera familiar del chico/a; Amigos y apoyo social (AMYAS), que en 6 ítems explora la naturaleza de las relaciones sociales del chico/a con otros; Entorno escolar (ENESC), la cual evalúa en 6 preguntas la percepción del chico/a sobre su aptitud para el aprendizaje, concentración y sensaciones sobre la escuela; Rechazo Social (RESOC), que en 3 ítems recoge los sentimientos de rechazo por parte de los compañeros; y Recursos económicos (RECON), la cual en 3 preguntas, evalúa la percepción sobre la capacidad financiera familiar. La adaptación a este cuestionario para población chilena puede ser solicitada al proyecto KIDSCREEN*. En la presente investigación, la Escala presentó un alfa de Cronbach de .81 para el cuestionario total, dato similar reportado a otras investigaciones en adolescentes chilenos ${ }^{18}$.

Las competencias parentales fueron evaluadas con la versión para hijos de la Escala de Competencia Parental Percibida (ECPP-h) ${ }^{19}$. Esta escala es autoadministrada y evalúa las aptitudes, conductas y competencias parentales de padres y madres percibidas por los hijos a través de 3 dimensiones: Implicancia Escolar (IE), Resolución de Conflictos (RC) y Consistencia Disciplinar (CD).

Una vez aprobado el proyecto por el Comité de ética de la Universidad Católica del Norte, se realizaron reuniones en los establecimientos con los padres/madres, a fin de explicarles el objetivo de la investigación y obtener la firma del consentimiento informado. Fueron aplicados 1130 cuestionarios en grupos de cursos, con un tiempo promedio de duración de 40 a 60 minutos. Los datos fueron ingresados a una base en SPSS versión 17. Se describen las medidas de tendencia central de todas las dimensiones que componen las escalas, tanto a nivel de la muestra total como separadas por sexo y tipo de establecimiento educacional.

http://www.kidscreen.de/cms/node/98 
Con excepción de la variable IE, ninguna de las otras variables tanto de competencia parental como de calidad de vida se distribuye normalmente ( $\mathrm{p}<0,05$ en prueba Kolmogorov-Smirnov). Pese a esto, se decidió aplicar igualmente la prueba t de student y ANOVA, cuyos análisis siguen siendo robustos pese al alejamiento de la normalidad, dado el tamaño muestral $^{20}$. Bajo este supuesto, para evaluar las diferencias, se utiliza la prueba t de student para grupos independientes en el caso del sexo y ANOVA con pruebas post hoc de Bonferroni para el tipo de establecimiento y rangos de edad. Finalmente, se correlacionaron los puntajes obtenidos en las distintas dimensiones evaluadas de CV con las competencias parentales a través del coeficiente de correlación de Pearson.

\section{Resultados}

Fueron encuestados 1130 sujetos entre 8 y 18 años de edad (media de 157,37 meses; $\mathrm{DE}=31,01)$. Con rango de edades que van entre los 8 a 10 años (25,7\%), 11 a 13 años (35,8\%), 14 a 16 años (32,7\%) y 17 a 18 años (5,8\%). Del total, 400 participantes asistían a establecimientos privados, 400 a establecimientos subvencionados (privados con aporte estatal) y 330 a establecimientos públicos. Se evaluó a 553 (48,9\%) hombres, con una media de edad de 159,59 meses (DE $=31,62)$ y 577 $(51,1 \%)$ mujeres, con una media de edad de $155,24$ meses ( $D E=30,29)$.

Al evaluar la calidad de vida por sexo, la media de los hombres es significativamente superior a la de las mujeres en BIFIS ( $\mathrm{t}=5,170$; $\mathrm{p}<0,05)$ y RESOC $(\mathrm{t}=3,758 ; \mathrm{p}<0,05)$. La media en AMYAS $(t=-3,843$; $p<0,05)$ y ENESC $(t=-2,642 ; p<0,05)$, es significativamente superior en las mujeres por sobre los hombres (tabla 1).

$\mathrm{Al}$ analizar por tipo de establecimiento educacional, se observa diferencias significativas en todas las dimensiones (tabla 2). La media de los menores de establecimientos subvencionados y privados es significativamente mayor que la media de los establecimientos públicos en siete de las dimensiones evaluadas. La media de los menores de establecimientos públicos es mayor que la media de los establecimientos privados y subvencionados en EANIM y RESOC.

La tabla 2, contiene las medias por rango de edad. Se encuentran diferencias significativas en todas las dimensiones de CV. Las medias del rango de 8 a 10 años son significativamente mayores a las de los otros rangos de edad en BIFIS, BIPSI, EANIM, ENESC y RESOC.

Tabla 1. Medias reportadas en las dimensiones de CV por sexo

\begin{tabular}{|c|c|c|c|}
\hline \multirow[b]{2}{*}{ Dimensiones } & \multicolumn{2}{|l|}{ Total } & Sexo \\
\hline & $\begin{array}{c}(x \pm D E) \\
n=1130\end{array}$ & $\begin{array}{c}\text { Hombres } \\
(x \pm D E) \\
n=553\end{array}$ & $\begin{array}{l}\text { Mujeres } \\
(x \pm D E) \\
n=577\end{array}$ \\
\hline Bienestar físico & $45,39 \pm 8,46$ & $46,70 \pm 8,35^{*}$ & $44,13 \pm 8,39$ \\
\hline Bienestar psicológico & $53,91 \pm 11,44$ & $53,76 \pm 11,48$ & $54,06 \pm 11,42$ \\
\hline Estado de ánimo & $23,08 \pm 10,55$ & $22,58 \pm 10,33$ & $23,56 \pm 10,74$ \\
\hline Autopercepción & $39,12 \pm 5,31$ & $38,91 \pm 5,42$ & $39,33 \pm 5,20$ \\
\hline Autonomía & $46,26 \pm 11,09$ & $46,80 \pm 10,61$ & $47,75 \pm 11,52$ \\
\hline Relación con los padres & $51,64 \pm 11,87$ & $51,90 \pm 11,44$ & $51,38 \pm 12,27$ \\
\hline Recursos económicos & $48,75 \pm 10,07$ & $48,31 \pm 10,03$ & $49,17 \pm 10,06$ \\
\hline Amigos y apoyo social & $56,82 \pm 11,13$ & $55,53 \pm 11,21$ & $58,06 \pm 10,91$ * \\
\hline Entorno escolar & $49,92 \pm 9,77$ & $49,14 \pm 9,57$ & $50,67 \pm 9,91$ * \\
\hline Rechazo social & $14,17 \pm 10,43$ & $15,35 \pm 10,57^{*}$ & $13,03 \pm 10,17$ \\
\hline
\end{tabular}


La tabla 4 muestra las medias por sexo y tipo de establecimiento en cuanto a competencias parentales. La media de los hombres es significativamente superior a la de las mujeres en la dimensiones $\mathrm{RC}(\mathrm{t}=4,634$ : $\mathrm{p}<0,05)$ y CD $(t=2,693: p<0,05)$. Al evaluar por tipo de establecimiento educacional, se observa diferencias significativas entre las dimensiones de IE y RC, en donde la media en implicancia escolar es mayor en los participantes de los establecimientos subvencionados por sobre la de los privados y públicos, en tanto,

Tabla 2. Medias reportadas en las dimensiones de CV por tipo de establecimiento

\begin{tabular}{|c|c|c|c|c|}
\hline \multirow[b]{2}{*}{ Dimensiones } & \multicolumn{4}{|c|}{ Tipo de Establecimiento } \\
\hline & $\begin{array}{l}\text { Público } \\
(x \pm D E) \\
n=400\end{array}$ & $\begin{array}{c}\text { Subvencionado } \\
(x \pm D E) \\
n=400\end{array}$ & $\begin{array}{l}\text { Privado } \\
(x \pm D E) \\
n=330\end{array}$ & ANOVA \\
\hline Bienestar físico & $43,44 \pm 8,60$ & $46,51 \pm 8,24^{a}$ & $46,38 \pm 8,27^{a}$ & $\left(F_{(2,1127)}=16,791 ; p<0,01\right)$ \\
\hline Bienestar psicológico & $51,37 \pm 12,11$ & $55,61 \pm 10,15^{a}$ & $54,94 \pm 11,58^{a}$ & $\left(F_{(2,1127)}=15,996 ; p<0,01\right)$ \\
\hline Estado de ánimo & $25,36 \pm 10,66^{b}$ & $21,50 \pm 9,71$ & $22,23 \pm 10,94$ & $\left(F_{(2,1127)}=15,279 ; p<0,001\right)$ \\
\hline Autopercepción & $39,07 \pm 5,78^{c}$ & $39,74 \pm 5,09 c$ & $38,44 \pm 4,89$ & $\left(\mathrm{~F}_{(2,1127)}=5,505 ; \mathrm{p}<0,05\right)$ \\
\hline Autonomía & $44,93 \pm 11,93$ & $47,42 \pm 10,17^{a}$ & $46,46 \pm 10,96^{a}$ & $\left(F_{(2,1127)}=5,146, p<0,05\right)$ \\
\hline Relación con los padres & $48,21 \pm 12,37$ & $55,03 \pm 10,20^{a}$ & $51,68 \pm 11,96^{a}$ & $\left(F_{(2,1127)}=35,018 ; p<0,01\right)$ \\
\hline Recursos económicos & $45,84 \pm 10,30$ & $51,13 \pm 9,21^{a}$ & $49,39 \pm 9,25^{a}$ & $\left(F_{(2,1127)}=30,012 ; p<0,01\right)$ \\
\hline Amigos y apoyo social & $53,41 \pm 12,04$ & $59,96 \pm 9,35^{a}$ & $57,16 \pm 10,80^{a}$ & $\left(F_{(2,1127)}=37,067 ; p<0,01\right)$ \\
\hline Entorno escolar & $47,63 \pm 10,54$ & $51,66 \pm 8,81^{a}$ & $50,59 \pm 9,40^{a}$ & $\left(F_{(2,1127)}=18,622 ; p<0,01\right)$ \\
\hline Rechazo social & $17,18 \pm 11,76^{\mathrm{b}}$ & $12,28 \pm 8,08$ & $12,18 \pm 9,53$ & $\left(F_{(2,1127)}=27,165 ; p<0,01\right)$ \\
\hline
\end{tabular}

aedia significativamente mayor que la de establecimientos públicos $(p<0,01)$; ${ }^{b}$ Media significativamente mayor que la de establecimientos privados y subvencionados $(p<0,01)$. cMedia significativamente mayor $(p<0,05)$ que establecimientos privados.

Tabla 3. Medias reportadas en las dimensiones de CV por rango de edad

\begin{tabular}{|c|c|c|c|c|c|}
\hline $\begin{array}{l}\text { Rango de edad } \\
\text { Dimensiones }\end{array}$ & $\begin{array}{c}(1) \\
8 \text { a } 10 \text { años } \\
(x \pm D E) \\
n=290\end{array}$ & $\begin{array}{c}(2) \\
11 \text { a } 13 \text { años } \\
(x \pm D E) \\
n=404\end{array}$ & $\begin{array}{c}(3) \\
14 \text { a } 16 \text { años } \\
(x \pm D E) \\
n=370\end{array}$ & $\begin{array}{c}(4) \\
17 \text { a } 18 \text { años } \\
(x \pm D E) \\
n=66\end{array}$ & ANOVA \\
\hline Bienestar físico & $47,72 \pm 8,38^{\mathrm{a}}$ & $45,91 \pm 8,36^{b}$ & $43,85 \pm 8,04^{c}$ & $40,56 \pm 8,25$ & $\left(F_{(3,1126)}=20,090 ; p<0,01\right)$ \\
\hline Bienestar psicológico & $56,90 \pm 10,23^{a}$ & $54,27 \pm 11,92$ & $52,36 \pm 10,74$ & $47,27 \pm 13,18^{d}$ & $\left(F_{(3,1126)}=17,093, p<0,01\right)$ \\
\hline Estado de ánimo & $20,15 \pm 10,89^{a}$ & $23,14 \pm 10,83$ & $24,23 \pm 9,30$ & $29,12 \pm 10,26^{e}$ & $\left(F_{(3,1126)}=16,772 ; p<0,01\right)$ \\
\hline Autopercepción & $38,24 \pm 6,31^{d}$ & $39,42 \pm 5,31$ & $39,53 \pm 4,54$ & $38,90 \pm 3,98$ & $\left(F_{(3,1126)}=3,843 ; p<0,01\right)$ \\
\hline Autonomía & $46,84 \pm 12,19$ & $46,09 \pm 11,03$ & $46,74 \pm 10,04$ & $42,05 \pm 11,34^{9}$ & $\left(F_{(3,1126)}=3,721 ; p<0,05\right)$ \\
\hline Relación con los padres & $54,04 \pm 11,69^{h}$ & $51,87 \pm 11,71$ & $50,87 \pm 11,32$ & $43,93 \pm 13,05^{\dagger}$ & $\left(F_{(3,1126)}=14,983 ; p<0,01\right)$ \\
\hline Recursos económicos & $46,38 \pm 11,05^{d}$ & $49,54 \pm 10,03$ & $50,31 \pm 8,95$ & $45,52 \pm 9,34^{d}$ & $\left(F_{(3,1126)}=11,675 ; p<0,01\right)$ \\
\hline Amigos y apoyo social & $55,27 \pm 12,26^{i}$ & $57,21 \pm 10,92$ & $58,36 \pm 9,97$ & $52,61 \pm 11,59^{d}$ & $\left(F_{(3,1126)}=7,685, p<0,01\right)$ \\
\hline Entorno escolar & $55,02 \pm 9,50^{\mathrm{a}}$ & $49,09 \pm 9,88$ & $48,30 \pm 8,27$ & $41,67 \pm 7,74^{d}$ & $\left(F_{(3,1126)}=52,706, p<0,01\right)$ \\
\hline $\begin{array}{l}\text { Aceptación social } \\
\text { (bullying) }\end{array}$ & $17,46 \pm 12,34^{a}$ & $14,35 \pm 10,14^{h}$ & $11,76 \pm 8,64$ & $12,10 \pm 8,14$ & $\left(F_{(3,1126)}=17,826, p<0,01\right)$. \\
\hline
\end{tabular}

${ }^{a}$ Media significativamente mayor que rango $2(p<0,01)$, $3(p<0,01)$ y $4(p<0,01)$; ${ }^{b}$ Media significativamente mayor que rango $3(p<0,01)$ y $4(p<0,01)$; 'Media significativamente mayor que rango $4(p<0,05)$; ${ }^{d}$ Media significativamente menor que rango $2(p<0,01)$ y $3(p<0,01)$; eMedia significativamente mayor que rango $1(p<0,01)$, $2(p<0,01)$ y $3(p<0,01)$; ${ }^{f}$ Media significativamente menor que rango $1(p<0,01), 2(p<0,01)$ y $3(p<0,01)$; 9Media significativamente menor que rango $1(p<0,01)$

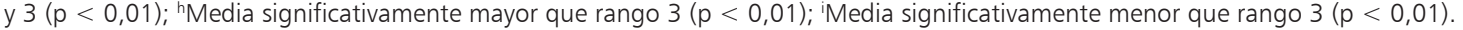


la media en resolución de conflictos es mayor en los establecimientos públicos, por sobre los otros dos tipos de establecimientos. Al comparar las medias por rangos de edad (tabla 5), se encuentran diferencias significativas entre las dimensiones, de IE y CD, en donde la media del rango de edad entre 8 y 10 años es significativamente mayor que la de los otros rangos de edad (tabla 5).

$\mathrm{Al}$ analizar la relación existente entre los puntajes obtenidos en las dimensiones de CV y las dimensiones de competencias parentales (tabla 6), se encuentra que la IE se correlaciona positivamente con BIFIS, BIPSI, AUTON, REPAD, RECON, AMYAS y ENESC, e inversamente con EANIM y con RESOC.

En lo que se refiere a la RC, se correlaciona positivamente con EANIM, APERC y RESOC, e inversamente con BIPSI, AUTON, REPAD y RECON. Con relación a la CD, está se correlaciona positivamente con BIFIS, BIPSI, APERC, AUTON, REPAD, RECON, AMYAS, ENESC y RESOC e inversamente con EANIM (tabla 6).

La tabla 7 muestra las correlaciones obtenidas entre las dimensiones de CV y las de competencias parentales por rango de edad. Tal como se observa, la IE se correlaciona positivamente en todos los rangos de edad con BIFIS, BIPSIC, AUTON, REPAD, RECON, AMYAS y ENESC, en tanto lo hace de manera inversa igualmente en todos los rangos de edad con el EANIM y RESOC, salvo en el rango de 17 a 18 años. La CD se correlaciona positivamente en todos los rangos de edad con BIFIS, BIPSI, REPAD y ENESC. Con excepción del rango de 17-18 años, también se correlaciona en todas las edades con la AUTON y AMYAS, y a excepción del tramo de 8 a 10 años, con RECON.

Tabla 4. Medias reportadas en las dimensiones de competencias parentales por sexo y tipo de establecimiento

\begin{tabular}{|c|c|c|c|c|c|c|c|}
\hline \multirow[b]{2}{*}{ Dimensión } & \multirow{2}{*}{$\begin{array}{c}\text { Total } \\
(x \pm D E)\end{array}$} & \multicolumn{2}{|c|}{ Sexo } & \multicolumn{4}{|c|}{ Establecimiento } \\
\hline & & $\begin{array}{l}\text { Hombres } \\
(x \pm D E) \\
n=553\end{array}$ & $\begin{array}{l}\text { Mujeres } \\
(x \pm D E) \\
n=577\end{array}$ & $\begin{array}{l}\text { Público (1) } \\
\text { (x } \pm \text { DE) } \\
n=400\end{array}$ & $\begin{array}{c}\text { Subvencio- } \\
\text { nado (2) } \\
(x \pm D E) \\
n=400\end{array}$ & $\begin{array}{l}\text { Privado (3) } \\
\text { (x } \pm \text { DE) } \\
n=330\end{array}$ & ANOVA \\
\hline $\begin{array}{l}\text { Implicancia } \\
\text { escolar }\end{array}$ & $2,88 \pm 0,45$ & $2,88 \pm 0,43$ & $2,89 \pm 0,46$ & $2,76 \pm 0,50$ & $3,00 \pm 0,37^{a, b}$ & $2,89 \pm 0,43^{a}$ & $\begin{array}{c}\left(F_{(2,1127)}=29,499 ;\right. \\
p<0,01)\end{array}$ \\
\hline $\begin{array}{l}\text { Resolución } \\
\text { de conflictos }\end{array}$ & $2,28 \pm 0,41$ & $2,34 \pm 0,43$ & $2,22 \pm 0,38$ & $2,37 \pm 046^{c}$ & $2,21 \pm 0,34$ & $2,24 \pm 0,40$ & $\begin{array}{c}\left(F_{(2,1127)}=16,620 ;\right. \\
\text { p } 0,01)\end{array}$ \\
\hline $\begin{array}{l}\text { Consistencia } \\
\text { disciplinar }\end{array}$ & $2,74 \pm 0,52$ & $2,79 \pm 0,53$ & $2,70 \pm 0,52$ & $2,71 \pm 0,55$ & $2,77 \pm 0,51$ & $2,75 \pm 0,51$ & \\
\hline
\end{tabular}

a Media significativamente mayor que establecimiento público $(p<0,01)$; ${ }^{b}$ Media significativamente mayor que establecimiento privado $(p<0,01)$; ' Media significativamente mayor que establecimiento privado $(p<0,01)$ y subvencionado $(p<0,01)$.

Tabla 5. Medias reportadas en las dimensiones de competencias parentales por rango de edad

\begin{tabular}{|c|c|c|c|c|c|}
\hline Dimensiones & $\begin{array}{c}8 \text { a } 10 \text { años } \\
(1) \\
(x \pm D E) \\
n=290\end{array}$ & $\begin{array}{c}11 \text { a } 13 \text { años } \\
(2) \\
(x \pm D E) \\
n=404\end{array}$ & $\begin{array}{c}14 \text { a } 16 \text { años } \\
(3) \\
(x \pm D E) \\
n=370\end{array}$ & $\begin{array}{c}17 \text { a } 18 \text { años } \\
(4) \\
(x \pm D E) \\
n=66\end{array}$ & ANOVA \\
\hline $\begin{array}{l}\text { Implicancia } \\
\text { escolar }\end{array}$ & $3,02 \pm 0,42^{\mathrm{a}}$ & $2,90 \pm 0,46$ & $2,82 \pm 0,41$ & $2,57 \pm 0,52^{b}$ & $\left(F_{(3,1126)}=22,953, p<0,01\right)$ \\
\hline $\begin{array}{l}\text { Resolución de } \\
\text { conflictos }\end{array}$ & $2,30 \pm 0,49$ & $2,28 \pm 0,42$ & $2,24 \pm 0,34$ & $2,35 \pm 0,34$ & \\
\hline $\begin{array}{l}\text { Consistencia } \\
\text { disciplinar }\end{array}$ & $2,88 \pm 0,55^{a}$ & $2,75 \pm 0,53^{c}$ & $2,67 \pm 0,47$ & $2,52 \pm 0,49$ & $\left(F_{(3,1126)}=12,723, p<0,01\right)$ \\
\hline
\end{tabular}


URZÚA A. y cols.

Tabla 6. Correlaciones dimensiones de CV y competencias parentales percibidas $(n=1130)$

\begin{tabular}{|llccc|}
\hline & & Implicancia escolar & Resolución conflictos & Consistencia disciplinar \\
\hline Bienestar físico & $r$ & $0,369^{* *}$ & 0,03 & $0,219^{* *}$ \\
\hline Bienestar psicológico & $r$ & $0,469^{* *}$ & $-0,062^{*}$ & $0,252^{* *}$ \\
\hline Estado de ánimo & $r$ & $-0,323^{* *}$ & $0,253^{* *}$ & $-0,088^{* *}$ \\
\hline Autopercepción & $r$ & 0,02 & $0,108^{* *}$ & $0,083^{* *}$ \\
\hline Autonomía & $r$ & $0,410^{* *}$ & $-0,065^{*}$ & $0,208^{* *}$ \\
\hline Relación con los padres & $r$ & $0,639^{* *}$ & $-0,132^{* *}$ & $0,293^{* *}$ \\
\hline Recursos económicos & $r$ & $0,338^{* *}$ & $-0,095^{* *}$ & $0,127^{* *}$ \\
\hline Amigos y apoyo social & $r$ & $0,408^{* *}$ & $-0,01$ & $0,199^{* *}$ \\
\hline Entorno escolar & $r$ & $0,465^{* *}$ & $-0,03$ & $0,278^{* *}$ \\
\hline Aceptación social (bullying) & $r$ & $-0,091^{* *}$ & $0,236^{* *}$ & $0,064^{*}$ \\
\hline
\end{tabular}

r: coeficiente de correlación de pearson; ${ }^{*}$ correlación significativa al 0,05, ** correlación significativa al 0,01.

Tabla 7. Correlaciones dimensiones de CV y competencias parentales percibidas divididos por rango de edad

\begin{tabular}{|c|c|c|c|c|c|c|c|c|c|c|c|c|c|}
\hline \multirow{2}{*}{\multicolumn{2}{|c|}{ Dimensiones }} & \multicolumn{3}{|c|}{$\begin{array}{c}8 \text { a } 10 \text { años } \\
n=290\end{array}$} & \multicolumn{3}{|c|}{$\begin{array}{c}11 \text { a } 13 \text { años } \\
n=404\end{array}$} & \multicolumn{3}{|c|}{$\begin{array}{c}14 \text { a } 16 \text { años } \\
n=370\end{array}$} & \multicolumn{3}{|c|}{$\begin{array}{c}17 \text { a } 18 \text { años } \\
n=66\end{array}$} \\
\hline & & $\begin{array}{l}\text { Impli- } \\
\text { cancia } \\
\text { escolar }\end{array}$ & $\begin{array}{l}\text { Reso- } \\
\text { lución } \\
\text { conflic- } \\
\text { tos }\end{array}$ & $\begin{array}{l}\text { Consis- } \\
\text { tencia } \\
\text { disci- } \\
\text { plinar }\end{array}$ & $\begin{array}{l}\text { Impli- } \\
\text { cancia } \\
\text { escolar }\end{array}$ & $\begin{array}{l}\text { Reso- } \\
\text { lución } \\
\text { conflic- } \\
\text { tos }\end{array}$ & $\begin{array}{l}\text { Consis- } \\
\text { tencia } \\
\text { disci- } \\
\text { plinar }\end{array}$ & $\begin{array}{l}\text { Impli- } \\
\text { cancia } \\
\text { escolar }\end{array}$ & $\begin{array}{l}\text { Reso- } \\
\text { lución } \\
\text { conflic- } \\
\text { tos }\end{array}$ & $\begin{array}{l}\text { Consis- } \\
\text { tencia } \\
\text { disci- } \\
\text { plinar }\end{array}$ & $\begin{array}{l}\text { Impli- } \\
\text { cancia } \\
\text { escolar }\end{array}$ & $\begin{array}{l}\text { Reso- } \\
\text { lución } \\
\text { conflic- } \\
\text { tos }\end{array}$ & $\begin{array}{c}\text { Consis- } \\
\text { tencia } \\
\text { disci- } \\
\text { plinar }\end{array}$ \\
\hline $\begin{array}{l}\text { Bienestar } \\
\text { físico }\end{array}$ & $r$ & $0,297^{\star \star}$ & 0,037 & $0,140^{*}$ & $0,364^{* *}$ & 0,046 & $0,211^{* *}$ & $0,292^{* *}$ & $-0,003$ & $0,187^{\star *}$ & $0,483^{* *}$ & $-0,043$ & $0,256^{*}$ \\
\hline $\begin{array}{l}\text { Bienestar } \\
\text { psicológico }\end{array}$ & $r$ & $0,384^{* *}$ & $-0,1$ & $0,155^{* *}$ & $0,417^{\star *}$ & $-0,06$ & $0,248^{* *}$ & $0,450^{* *}$ & $-0,06$ & $0,232^{* *}$ & $0,695^{* *}$ & $-0,04$ & $0,317^{* *}$ \\
\hline $\begin{array}{l}\text { Estado de } \\
\text { ánimo }\end{array}$ & $r$ & $-0,203^{* *}$ & $0,282^{* *}$ & 0,02 & $-0,328^{* *}$ & $0,244^{* *}$ & $-0,08$ & $-0,262^{* \star}$ & $0,254^{\star *}$ & $-0,08$ & $-0,507^{\star *}$ & $0,305^{*}$ & $-0,12$ \\
\hline $\begin{array}{l}\text { Autopercep- } \\
\text { ción }\end{array}$ & $r$ & 0,05 & $0,161^{* *}$ & $0,117^{\star}$ & 0,02 & 0,06 & 0,05 & 0,07 & $0,109^{*}$ & $0,149^{* \star}$ & $-0,18$ & 0,22 & 0,1 \\
\hline Autonomía & $r$ & $0,435^{\star *}$ & $-0,03$ & $0,189^{* *}$ & $0,472^{* *}$ & $-0,07$ & $0,248^{* *}$ & $0,327^{\star *}$ & $-0,1$ & $0,178^{* *}$ & $0,299^{*}$ & $-0,01$ & 0,08 \\
\hline $\begin{array}{l}\text { Relación con } \\
\text { los padres }\end{array}$ & $r$ & $0,491^{\star *}$ & $-0,146^{*}$ & $0,155^{* *}$ & $0,663^{* *}$ & $-0,117^{\star}$ & $0,328^{* *}$ & $0,643^{* *}$ & $-0,134^{*}$ & $0,279^{* \star}$ & $0,790^{* *}$ & $-0,19$ & $0,411^{* *}$ \\
\hline $\begin{array}{l}\text { Recursos } \\
\text { económicos }\end{array}$ & $r$ & $0,351^{* *}$ & 0,02 & 0,07 & $0,359^{* *}$ & $-0,146^{* *}$ & $0,214^{* *}$ & $0,364^{* *}$ & $-0,130^{*}$ & $0,122^{*}$ & $0,508^{* *}$ & $-0,12$ & $0,255^{*}$ \\
\hline $\begin{array}{l}\text { Amigos y } \\
\text { apoyo social }\end{array}$ & $r$ & $0,484^{* *}$ & 0,08 & $0,202^{* *}$ & $0,389^{* *}$ & $-0,133^{* *}$ & $0,200^{* *}$ & $0,417^{\star *}$ & 0,08 & $0,242^{* *}$ & $0,456^{* *}$ & 0,14 & 0,18 \\
\hline $\begin{array}{l}\text { Entorno } \\
\text { escolar }\end{array}$ & $r$ & $0,378^{* *}$ & $-0,05$ & $0,198^{* *}$ & $0,433^{* *}$ & $-0,05$ & $0,248^{* *}$ & $0,447^{\star \star}$ & $-0,01$ & $0,239^{* *}$ & $0,462^{* *}$ & 0,1 & $0,323^{* *}$ \\
\hline $\begin{array}{l}\text { Aceptación } \\
\text { social } \\
\text { (bullying) }\end{array}$ & $r$ & $-0,167^{* *}$ & $0,275^{* *}$ & 0,07 & $-0,142^{* *}$ & $0,265^{* *}$ & 0,05 & $-0,113^{*}$ & $0,148^{* *}$ & 0 & $-0,17$ & 0,04 & $-0,24$ \\
\hline
\end{tabular}

r: coeficiente de correlación de pearson; *correlación significativa al 0,05; ${ }^{* *}$ correlación significativa al 0,01. 
La RC se encuentra relacionada positivamente en todos los rangos de edad con el EANIM. Con excepción del rango de 17-18 años, también se correlaciona positivamente en las otras edades con RESOC e inversamente con REPAD (tabla 7).

\section{Discusión}

Los resultados encontrados en la evaluación de la CV refuerzan los hallazgos ya reportados en otras investigaciones. Los hombres tienen una media mayor en bienestar físico, en tanto las mujeres la tienen en la dimensión de amigos y apoyo social ${ }^{21}$. Esto es esperable ya que las mujeres dan mayor prioridad a actividades destinadas a la socialización, mientras que los hombres dedican su tiempo de ocio a desarrollar actividades físicas y deportivas. De manera similar al estudio español de género y salud $^{21}$, los hombres autoreportaron una mejor relación con los padres que las mujeres, en tanto las mujeres una mejor relación que los hombres con el entorno escolar.

Se suma evidencia a la desigualdad en la CV dado el tipo de establecimiento educacional $^{13,22}$. Los establecimientos públicos reportan una puntuación inferior en la mayoría de las dimensiones de $\mathrm{CV}$, exceptuando las dimensiones de estado de ánimo y aceptación social. Esto podría deberse a varios factores, como un menor acceso a recursos materiales o menores niveles educativos de sus padres, en comparación a los alumnos que asistían a colegios privados, los cuales tienen mayor acceso a recursos materiales, de salud, etc ${ }^{13}$.

En relación a la CV y la edad, de manera similar a lo encontrado en otros estudios ${ }^{13,23}$, se encuentra que a medida que esta avanza la edad disminuye la media de la CV en la mayoría de las dimensiones. Los menores perciben de mejor manera su apariencia física que los/as adolescentes, ya que aún no llegan al período evolutivo de la pubertad, donde se producen cambios físicos notorios con la aparición de las características sexuales secundarias. Además la adolescencia es el período de transición social y psicológica entre la niñez y la adultez, lo que generaría cambios importantes tanto a nivel psicológico, social y escolar ${ }^{23}$. Desde un punto de vista psicosocial, los menores invierten más tiempo en actividades escolares y tienen una mejor relación con sus padres, ya que aún son dependientes de ellos, lo cual no ocurre con los adolescentes, debido a la tensión entre la dependencia y la necesidad de desprenderse y ser reconocidos por otros como un individuo con identidad propia ${ }^{24}$.

Con relación a las competencias parentales, los hombres perciben una mayor consistencia disciplinar y resolución de conflictos en sus padres que las mujeres, en tanto las mujeres perciben una mayor implicancia escolar de sus padres que los hombres. Es posible que esta diferencia se halle en estilos de crianza modelados por el sexo del menor. Así, los padres podrían tender a tener conductas de mayor control y disciplina con los varones, en tanto que con las mujeres, quienes teóricamente tendrían menos problemas conductuales o necesidad de mayor disciplina, podrían centrar su accionar en priorizar formas de interacción centradas en el ambiente escolar. Sobre este punto tampoco ha sido posible hallar evidencias empíricas que aporten mayor evidencia al respecto.

Un hallazgo interesante son las diferencias en las competencias parentales percibidas dado el tipo de establecimiento educacional al que asiste el menor. Los menores de establecimientos públicos perciben una menor implicancia escolar y consistencia disciplinar de sus padres. Posiblemente, el grado de implicancia de los padres en la educación de sus hijos esté vinculado al nivel educacional alcanzado por los padres, al tipo de relación existente entre padres e hijos/as, o al tipo de desarrollo de las competencias parentales, las cuales podrían estar disminuidas en los padres pertenecientes a sectores más deprivados socialmente. Este hecho es importante, ya que la evidencia indica que un involucramiento parental a nivel escolar juega un papel clave en la formación, obteniéndose beneficios tanto para los padres como para el niño: mejora la autoestima, ayuda a los padres a desarrollar actitudes positivas hacia el establecimiento educacional, disminuye la deserción, mejora las actitudes y conducta del alumno/a y favorece una comunicación positiva padre-hijo ${ }^{25}$.

En cuanto a la consistencia disciplinar, no 
se tiene conocimientos de estudios que avalen nuestros hallazgos pero si consideramos relevante mencionar que la disciplina podría derivar en resultados emocionales y sociales, tales como el mejoramiento conductual, control de impulsos y atención. Las herramientas y estrategias de los padres que buscan imponer un tipo de disciplina rígida pueden afectar negativamente el aspecto emocional y el desempeño social de los niños, en relación a dificultar su adquisición de habilidades para desarrollar relaciones sociales sostenibles ${ }^{26}$.

$\mathrm{Al}$ relacionar las percepciones de los menores sobre las competencias de sus padres con su propia evaluación de calidad de vida, se encontró que la implicancia escolar de los padres estaría relacionada positivamente con la mayoría de las dimensiones de CV evaluadas. Evidentemente, la implicación parental en asuntos escolares de niños/as y adolescentes promueve el éxito escolar y es percibido por los menores como positivo en cuanto su evaluación de bienestar. Paralelamente, se observa una relación inversa de esta dimensión con el dominio de CV de estado de ánimo, posiblemente debido a la presión que puede implicar para el menor un adecuado desempeño académico o las continuas exigencias escolares, que dada la implicancia de los padres, pueden "atentar" contra lo que los niños esperan hacer de su tiempo libre, en términos de ocio y recreación.

La consistencia disciplinar se encuentra relacionada, a excepción del estado de ánimo, de manera directa con todas las dimensiones de la CV. Como se mencionó, los padres y las madres son proveedores de la primera fuente de apoyo y consistencia para sus hijos e hijas; esto supone numerosas ventajas para su desarrollo, ya que está asociado con mejor salud física, salud mental, adaptación al estrés, mejor funcionamiento escolar, más alta autoestima mejor auto representación, creencia en la propia competencia, menos problemas de comportamiento y de delincuencia; además, puede amortiguar los efectos negativos del conflicto en las relaciones padres-hijos. Al mismo tiempo, está relacionado con mejores relaciones entre iguales, ya que de esta forma los niños/as adquieren habilidades acerca de cómo apoyar a otros, desarrollan un estilo de apego seguro, lo que les capacita para confiar y ser más cercanos $^{27}$.

En cuanto a la resolución de conflictos, una mayor percepción de ésta estaría relacionada con un mejor estado de ánimo, autopercepción y aceptación social. Esto se podría deber a que una adecuada manera de los padres para resolver problemas al interior de la familia, tiene una función en el desarrollo social, educativo, intelectual, afectivo y emocional en la población infanto juvenil, que no sólo es clave, sino que resulta básica para el desarrollo del individuo. Estudios existentes avalan los hallazgos de nuestra investigación ya que una solución de los conflictos por la vía positiva fomenta la evolución personal y permite que tanto el hijo/a como el padre lleguen a su máximo potencial. Esto quiere decir que los niño/as y adolescentes adquieren habilidades comunicacionales, una mejor autonomía y especialmente un mejor nivel en la relación con sus padres $^{28}$. Una adecuada resolución de conflictos parental, permite que se genere un vínculo emocional mutuo y consistente entre padres e hijos, lo que a su vez se traduce en una conexión de confianza que posibilita establecer el desarrollo emocional, protección, apertura en la comunicación y estímulo ${ }^{29}$.

En cuanto a las limitaciones del estudio, cabe mencionar la compartida por muchos investigadores: el déficit de investigaciones latinoamericanas en cuanto al desarrollo de instrumentos validados que permitan medir y evaluar las competencias parentales. En este caso el instrumento aplicado fue desarrollado y evaluado en población europea, especialmente la española. Como sugerencia para futuras investigaciones en el área, sería importante indicar a quién evaluará el niño/a y adolescente, puesto que los estilos parentales percibidos pueden para ambos padres, por separados o para un cuidador/a principal. Sería importante asimismo analizar el efecto que pudiesen tener otras variables en esta relación, por ejemplo el número de hermanos, las persona con la que viven los menores, el estado de salud, educación de los padres, cesantía, rango de ingreso, entre otros, que no pudieron ser incorporados en este estudio dada la extensión del cuestionario. 
Los datos encontrados en este estudio nos permiten aportar evidencia a la existencia del vínculo entre las competencias parentales y la $\mathrm{CV}$, sin embargo, no fue posible comprobar nuestra hipótesis de que a mayor grado de competencias parentales se encontraría una mayor $\mathrm{CV}$, puesto que existen diferencias dependiendo el tipo de competencia, encontrándose que en algunas competencias una relación inversa con CV.

Esta investigación pretende contribuir a futuras exploraciones en el área, específicamente en las líneas de acción que se pueden tomar a partir de los resultados obtenidos. Los datos recogidos avalan la necesidad de generar intervenciones dirigidas a la promoción y prevención, al diseño e implementación de programas orientados a que los padres puedan adquirir y/o fortalecer estrategias y herramientas que le permiten apoyar de la mejor forma la crianza y desarrollo de sus hijos/as. Creemos que esta investigación suma evidencia científica al conocimiento empírico de que las influencias que ejercen las prácticas parentales tienen un efecto directo en los resultados específicos del desarrollo, promueven un adecuado desarrollo de la autoestima, autonomía y felicidad de los hijos/as ${ }^{21}$.

\section{Referencias}

1.- $\quad$ The WHOQOL Group: The World Health Organization Quality of Life Assessment (WHOQOL). Position Paper from the World Health Organization. Soc Sci Med 2005; 4: 1403-9.

2.- Quinceno J, Vinaccia S: Calidad de vida relacionada con la salud infantil: Una aproximación conceptual. Psicología y Salud 2008; 18: 37-44.

3.- Rajmil L, Roizen M, Urzúa A: Calidad de vida y salud en la infancia y la adolescencia. Revista Típica 2010; 6: 244-9.

4.- Méndez Rubio I. Lázaro de Mercado P, Carbonell Estrany X, Figueras Aloy J: Calidad de vida en lactantes nacidos prematuros según ingresos por infección respiratoria. An Pediatr (Barc) 2010; 73: 121-31.

5.- Rajmil L, Estrada MD, Herdman M, et al: Concordancia entre padres e hijos en la calidad de vida relacionada con la salud en niños con trastorno por déficit de atención con hiperactividad: estudio longitudinal. An Pediatr (Barc) 2009; 70: 553-61.
6.- Kuczynski E, Silva CAA, Cristófani LM, Kiss MHB, Odone Filho V, Assumpção FB: Evaluación de la calidad de vida en niños y adolescentes portadores de enfermedades crónicas y/o incapacitadoras: un estudio brasileño. An Pediatr (Barc) 2003; 58: 550-5.

7.- Sardón Prado O, Morera G, Herdman M, et al: Versión española del TAPQOL: calidad de vida relacionada con la salud en niños de 3 meses a 5 años. An Pediatr (Barc) 2008; 68:420-4

8.- Rajmil L, Serra-Sutton V, Fernández-López JA, et al: Versión española del cuestionario alemán de calidad de vida relacionada con la salud en población infantil y de adolescentes: el Kindl. An Pediatr (Barc) 2004; 60: 514-21.

9.- González de Dios J: Calidad de vida relacionada con la salud: conocer e implementar en la toma de decisiones basada en pruebas en pediatría. An Pediatr (Barc) 2004; 60: 507-13.

10.- Urzúa A: Calidad de vida relacionada con la salud: Elementos conceptuales. Rev Med Chile 2010; 138: 358-65.

11.- Urzúa A, Mercado G: La evaluación de la calidad de vida de los y las adolescentes a través del KIDDOKINDL. Terapia Psicológica 2008; 26: 133-41.

12.- Detmar S, Bruil J, Ravens-Sieberer U, Gosch A, Bisegger $C$ : The use of focus groups in the development of the KIDSCREEN HRQL questionnaire. Quality of Life Research 2006; 15: 1345-53.

13.- Urzúa A, Cortés E, Vega S, Prieto L, Tapia K: Autoreporte de la Calidad de Vida en Niños y Adolescentes Escolarizados. Rev Chil Pediatr 2009; 80: 238-44.

14.- Barudy J: La Integración Escolar Como Parte de un Modelo de Protección Infantil Basado en la Promoción de la Resiliencia. I jornades "menors en edat escolar: conflictes i oportunitats”, 2005 noviembre, Palma de Mallorca, España.

15.- Rodrigo MJ, Máiquez ML, Martín J: Preservación Familiar: un enfoque positivo para la intervención con familias. 2008. Madrid: Editorial Pirámide.

16.- Rajmil L, Alonso X, Berra S, et al: Use of a children questionnaire of health-related quality of life (KIDSCREEN) as a measure of needs for health care services. J Adolesc Health 2006; 38: 511-8.

17.- Tebe C, Berra D, Herdman M, Aymerich M, Jordi A, Rajmil $L$ : Fiabilidad y validez de la versión española del KIDSCREEN-52 para población infantil y adolescente. Med Clin 2007; 17: 650-4.

18.- Urzúa A, Avendaño F, Díaz S, Checura D: Calidad de Vida y conductas alimentarias en la preadolescencia. Rev Chil Nutr 2010; 37: 282-92. 
19.- Bayot A, Hernández J: Evaluación de la competencia parental (ECPP). 2008. Madrid: Editorial Cepe.

20.- Montilla JM, Kromre, J: Robustez de las pruebas T en comparación de medias, ante violación de supuestos de normalidad y homocedasticidad. Rev Ciencia e Ing 2010; 31: 101-8.

21.- Vélez $R$, López $S$, Rajmil L: Género y salud percibida en la infancia y la adolescencia en España. Gaceta Sanitaria 2009; 23: 433-9.

22.- Rajmil L, Estrad M, Herdman M, Serra-Sutton V, Alonso $J$ : Calidad de vida relacionada con la salud (CVRS) en la infancia y la adolescencia: revisión de la bibliografía y de los instrumentos adaptados en España. Gaceta Sanitaria 2001: 15: 34-43.

23.- Bisegger C, Cloetta B, Von Rueden U, Abel T, RavensSieberer $U$ \& The European KIDSCREEN group: Health related quality of life: gender differences in childhood and adolescence. Soz.-Präventivmed 2005; 50: 281-91.
24.- Papalia D, Olds S, Feldman R: Psicología del Desarrollo, de la infancia a la adolescencia. 2005. México. Mc Graw Hill.

25.- Sánchez P: Discapacidad, familia y logro escolar. Rev Iberoam Ed 2006; 40: 1-10.

26.- Dillon B: Programas de apoyo parental y resultados en los niños. Enciclopedia sobre el Desarrollo de la Primera Infancia. 2010, Centre of Excellence for Early Childhood Development, disponible en http://www. enciclopedia-infantes.com/pages/PDF/habilidades_parentales.pdf

27.- Gardner KA, Cutrona CE: Social support communication in families. Vangelisti, Handbook of family communication 2004; 495-512.

28.- Megías E, Elzo J, Rodríguez E, et al: Comunicación y conflictos entre hijos y padres. Fundación de Ayuda contra la Drogadicción. 2003. Madrid.

29.- Montalbo G: Conexión es Protección. Edu Salud Informa, División de Madres, niños y adolescentes 2005; 1: 1-4. 